\title{
inventio
}

La génesis de la cultura universitaria en Morelos

Año 15, núm. 37, noviembre 2019

ISSN: 2007-1760 (impreso) 2448-9026 (digital) | DOI: 10.30973/inventio/2019.15.37/8

CIENCIA Y TECNOLOGÍA

\section{Valores hematológicos del guacamayo híbrido de Santa Fe (Primolius sp.)}

\section{Antonio Alejandro Sciabarrasi}

ORCID: 0000-0002-0601-3166/asciabarrasi@fcv.unl.edu.ar

Profesor-investigador, Cátedra de Zoología, Diversidad y Ambiente, Facultad de Ciencias Veterinarias (FCV),

Universidad Nacional del Litoral (UNL)/Estación Biológica La Esmeralda

\section{Marcelo Ruiz}

Profesor-investigador, Laboratorio de Análisis Clínico, FCV, UNL

\section{Leandro Neme}

Personal técnico, Centro de Medicina Comparada, Instituto de Ciencias Veterinarias del Litoral (ICIVET), FCV, UNL

\section{RESUMEN}

El guacamayo de Santa Fe (Primolius sp.) se considera un nuevo loro híbrido del nuevo mundo recientemente descrito en la avicultura. Para establecer rangos hematológicos de referencia, se tomaron muestras de sangre de dos especímenes (un macho y una hembra) criados en cautividad en la Estación de Rescate, Rehabilitación y Reubicación de Fauna La Esmeralda, en Santa Fe, Argentina. El promedio de eritrocitos y leucocitos fue $2705 \times 10^{3} / \mathrm{ml}$ y de $11.10 \times 10^{3} / \mathrm{ml}$, respectivamente; hematocrito, $52 \%$ y $200 \mathrm{fl}$ de volumen corpuscular medio; recuento diferencial leucocitario, $65.61 \%$ de heterófilos y $26.41 \%$ para los linfocitos. El diámetro promedio de los eritrocitos fue de $11.7 \mu \mathrm{m}$. Estos resultados podrían emplearse como valores locales de referencia para este grupo de aves.

\section{PALABRAS CLAVE}

guacamayo Santa Fe; Primolius sp.; Psittacidae; hematología; valores normales

Universidad Autónoma del Estado de Morelos / Secretaría Académica

Dirección de Publicaciones y Divulgación

inventio.uaem.mx, inventio@uaem.mx 


\section{Introducción}

Los guacamayos, guacamayas, parabas o lapas son un grupo de aves de la familia de los loros (Psittacidae), del orden de los Psittaciformes, que engloba a diecisiete especies, las cuales están distribuidas en seis géneros, todos de origen americano y que habitan desde las selvas de México hasta el noreste de Argentina, en general. De estos géneros, el Primolius agrupa a tres especies, que son Primolius maracana, Primolius couloni y Primolius auricollis (Jordan, 2009).

Primolius híbrido pertenece también a la familia de las Psittacidae y se le conoce localmente como guacamayo Santa Fe (figura 2). Su distribución no existe en la naturaleza debido a que es un híbrido de cautiverio que se originó del cruzamiento de Primolius maracana por Primolius auricollis, en la Estación de Rescate, Rehabilitación y Reubicación de Fauna La Esmeralda (Sciabarrasi \& Neme, 2018).

El tamaño promedio del guacamayo Santa Fe es de $41 \mathrm{~cm}$ y su peso varía entre 207 y $294 \mathrm{~g}$. El plumaje es predominantemente verde, con verde rojizo en las partes ventrales; la cabeza, roja, negra y azul con una máscara facial blanca. No existe dimorfismo sexual evidente entre machos y hembras. Los adultos poseen el iris amarillo y patas rosadas, mientras los juveniles presentan iris oscuro y patas grises (Sciabarrasi \& Neme, 2018). De acuerdo con su condición de híbridos, no integran ninguna legislación vigente local, nacional o internacional.

La hematología clínica ha sido por mucho tiempo uno de los criterios más empleados como medida de diagnóstico de la salud (Tell, Kabbur, Smith, Dahl \& Cullor, 1997; Feldman \& Synk, 2006), pues, además, permite evaluar cambios resultantes de demandas fisiológicas; por ejemplo, en las aves, el número de células rojas varía según la edad, el género, hora del día, temporada del año, altitud, nutrición y niveles circulantes de hormonas como andrógenos, estrógenos y tiroideas. Además, el estudio hematológico puede ser de gran utilidad para el conocimiento de la adaptación de las especies al medio ambiente y para el manejo de la fauna con fines de conservación de la diversidad biológica.

Tanto en uno como en otro caso, la interpretación de los resultados pasa por la determinación de la normalidad de los datos obtenidos y, en su caso, del grado de desviación que presenta un parámetro frente a lo esperado. De ahí la importancia de disponer de datos normales o de referencia para la especie, a fin de poder interpretar los valores obtenidos en un determinado individuo (Soto Piñeiro, Acosta Guevara \& Cruz López, 2009).

Debido a que existe poca información sobre estos híbridos, en el presente estudio se establecen los valores hematológicos normales de Primolius sp. híbrido (guacamayo Santa Fe) en condiciones de cautiverio.

\section{Materiales y métodos}

El trabajo se realizó en la Estación de Rescate, Rehabilitación y Reubicación de Fauna La Esmeralda, durante julio de 2018. Se utilizaron dos guacamayos Santa Fe adultos, una hem- 
bra y un macho. Las aves estaban clínicamente sanas y con pesos de $270 \mathrm{~g}$ para la hembra y $350 \mathrm{~g}$ para el macho (figura 1). Recibían una dieta balanceada de frutas, vegetales y semillas.

Figura 1

Pareja de guacamayo Santa Fe híbrido

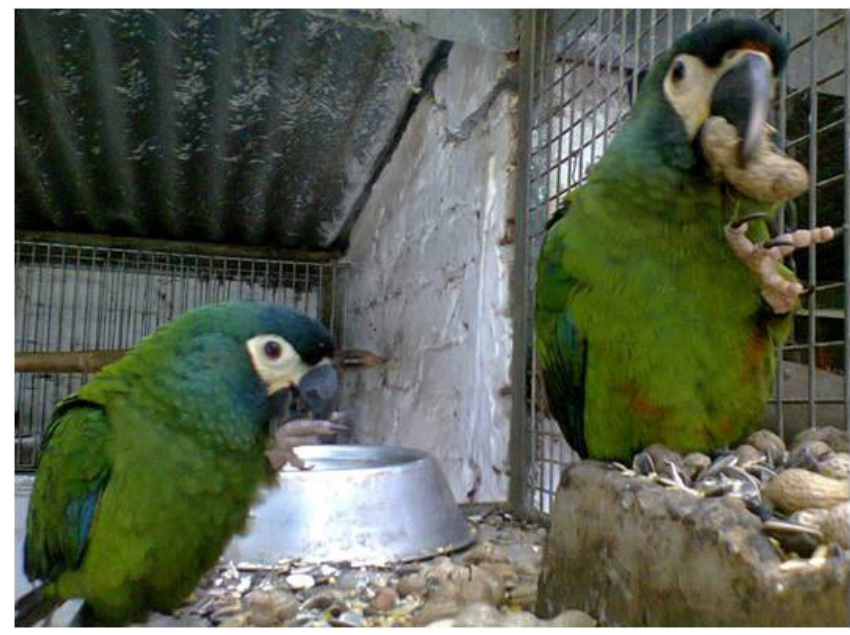

Foto: Antonio Sciabarrasi

Figura 2

Extracción de sangre de las venas yugular (A) y braquial (B) en pareja de guacamayo Santa Fe híbrido
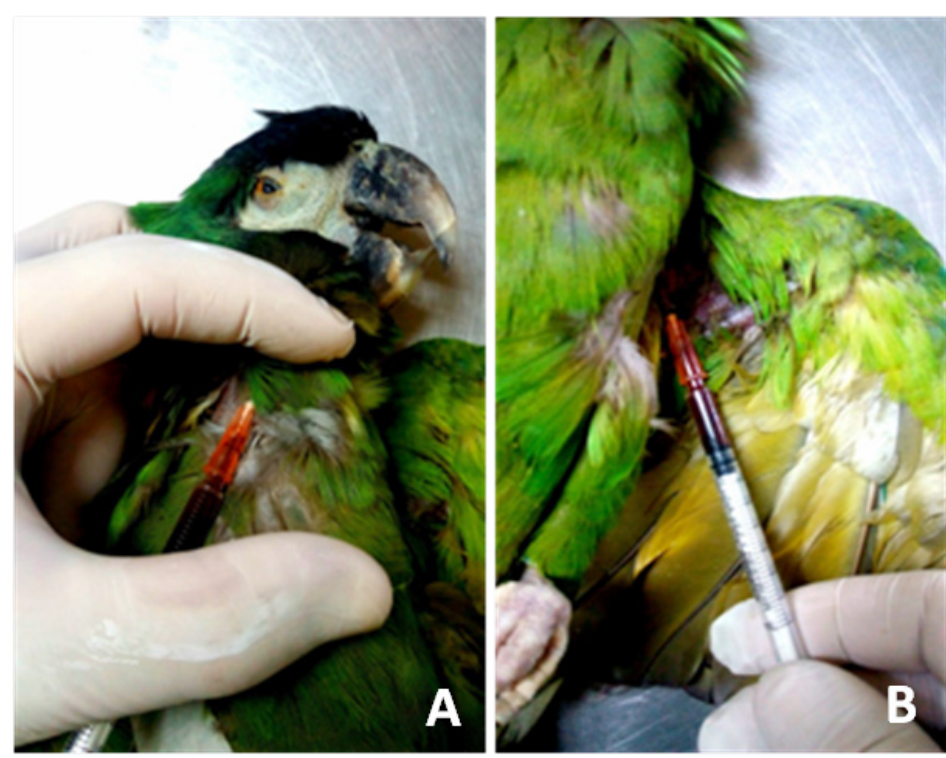

Foto: Antonio Sciabarrasi

Año 15, núm. 37, noviembre 2019

ISSN: 2007-1760 (impreso) 2448-9026 (digital) | DoI: 10.30973/inventio/2019.15.37/8 
La captura fue mediante contención física (Aguilar, Hernández \& Hernández, 2005), con una red con mango de metal a modo de copo. Se colocaron individualmente en un recipiente transparente de plástico y se los pesó. Se posicionó al psitácido en decúbito dorsal y, con una aguja $\mathrm{N}^{\circ}{ }^{25} / 8$, se extrajo $1 \mathrm{ml}$ total de sangre de las venas braquial y yugular. Parte de la sangre (0.5 ml) se transfirió a un tubo con heparina de litio para el análisis hematológico (glóbulos rojos y blancos, hematocrito y volumen corpuscular medio [VCM]), y con el $0.5 \mathrm{ml}$ restante se prepararon dos frotis por individuo, uno de ellos para la observación citomorfológica, y el último, para el recuento diferencial de leucocitos.

En el recuento de glóbulos blancos y glóbulos rojos se siguió el protocolo de Natt y Herrick (1952). Para el valor del VCM se empleó la fórmula (Hematocrito, en \% X 10)/N. e eritrocitos, en millones/ml. Para el recuento diferencial de leucocitos, los frotis se colorearon con Wright (pH 6.8) y se observaron con un microscopio de campo claro.

Los frotis para la observación citomorfológica se dejaron secar a temperatura ambiente, se fijaron en metanol absoluto a $8{ }^{\circ} \mathrm{C}$ por 20 min y se tiñeron con los colorantes Giemsa, MayGrunwald, Wright, hematoxilina-eosina y nitrato de plata. Se utilizó un ocular micrométrico incorporado al microscopio para la biometría de las células sanguíneas.

Para la determinación de los promedios y dispersión de los parámetros hematológicos, se utilizaron las medidas descriptivas, empleando la media aritmética como medida de tendencia central y la desviación estándar como medida de dispersión.

Tabla 1

Valores promedio de las series eritrocíticas y heterocíticas de dos guacamayos

Santa Fe híbridos adultos (Primolius sp.)

\begin{tabular}{|c|c|c|c|}
\hline \multicolumn{2}{|c|}{ Serie eritrocífica } & \multicolumn{2}{|c|}{ Serie leucocítica } \\
\hline Variables & Promedios & Variables & Promedios \\
\hline Eritrocitos $\left(\mathrm{x} 10^{3} / \mathrm{ml}\right)$ & 2705 & Leucocitos $\left(\times 10^{3} / \mathrm{ml}\right)$ & 11.10 \\
\hline Hematocrito (\%) & 52 & Heterófilos (\%) & 65.61 \\
\hline \multirow[t]{4}{*}{ VMC (ft) } & 200 & Linfocitos (\%) & 26.41 \\
\hline & & Monocitos (\%) & 4.68 \\
\hline & & Eosinófilos (\%) & 2.30 \\
\hline & & Basófilos (\%) & 1 \\
\hline
\end{tabular}

Tabla 2

Promedio de células sanguíneas de dos guacamayos Santa Fe híbridos adultos (Primolius sp.)

\begin{tabular}{cc}
\hline Célula & Diámetro $(\boldsymbol{\mu m})$ \\
\hline Eritrocito & 11.7 \\
\hline Heterófilo & 10.8 \\
\hline Linfocito & 7.4 \\
\hline Basófilo & 8.0 \\
\hline Monocito & 8.8 \\
\hline
\end{tabular}

Año 15, núm. 37, noviembre 2019 


\section{Resultados}

Los valores hematológicos promedio se muestran en la tabla 1 y el tamaño de las células sanguíneas en la tabla 2.

Los eritrocitos se observaron como células de morfología elíptica, cuyos extremos están ligeramente redondeados; contienen un citoplasma acidófilo, sin granulaciones en citoplasma. El núcleo presenta una forma redondeada, con cromatina homogénea y granular (figura 3a). El citoplasma es rosáceo y el núcleo es rojo.

Los heterófilos son considerados "la ventana del estado de salud del ave" (McDonald, 1996). Tienen forma redondeada y presentan un núcleo segmentado hasta con tres lobulaciones bien definidas, con pequeñas granulaciones violeta oscuro que enmascaran el citoplasma azul celeste o rosado y finamente granulado (figura 3b). Las células basófilas poseen una forma redondeada con un citoplasma con granulaciones más prominentes (figura 3c). Los monocitos presentaron un núcleo grande de forma irregular con granulaciones, citoplasma compacto y homogéneo sin granulaciones (figura 3d).

Los linfocitos fueron esféricos, de tamaño diverso. Algunos poseen un núcleo más eucromático que los heterófilos, y ocupan todo el citoplasma de la célula (figura ze) (Solari, David, Zoila, Rafael \& José, 2011).

\section{Figura 3}

\section{Morfología sanguínea del guacamayo Santa Fe, Primolius sp. híbrido}

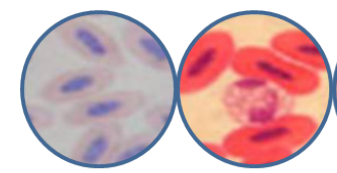

(a) (b)

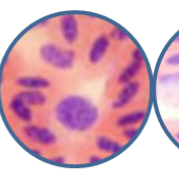

(c)

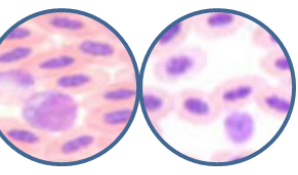

(d)

(e)

Fuente: Coloración May-Grunwald Giemsa. Aumento 100X. Foto: Marcelo Ruiz

\section{Discusión y conclusiones}

En las aves, el tamaño de los eritrocitos oscila entre 11 y 16 micras para el diámetro mayor y de 6 a 10 micras para el diámetro menor (Bergman, 1957). En el caso de Primolius sp. híbrido, los valores obtenidos se encuentran dentro de los límites descritos.

El número de eritrocitos en las aves varía entre 1.5 y $6.6 \times 10^{6} / \mathrm{ml}$, y es inversamente proporcional al peso del ave, esto es, aves con mayor peso tienen menor cantidad de glóbulos rojos (Bergman, 1957). Por ejemplo, el colibrí rubí (Chrysolampis mosquitus) tiene un peso de 2-4 $\mathrm{g}$ y un promedio de $6.59 \times 10^{6} / \mathrm{ml}$, en tanto que el avestruz (Struthio camelos) tiene un pe-

Año 15, núm. 37, noviembre 2019 
so de $120 \mathrm{~kg}$ y sólo $1.9 \times 10^{6} / \mathrm{ml}$ (Saint-Girons \& Duguy, 1963). El Primolius sp. híbrido es un ave de tamaño intermedio y presentó un promedio de $2705 \times 10^{3} / \mathrm{ml}$.

En psitácidos, los valores de hematocrito se encuentran entre 37\% y 53\% (Saint-Girons y Duguy, 1963), y en el caso de Primolius sp. híbrido fue de 52\%. Asimismo, los valores leucocitarios en la mayoría de los psitácidos varían entre 8 a $15 \times 10^{3} / \mathrm{ml}$ (Cruz Alvarado, Arraga-Alvarado, Rincón Rincon, Fernández, Aguilar Lara, Villasmil-Ontiveros, Gómez \& Henriquez, 2008), $y$ en este caso estuvo dentro de esos valores.

Mc Donald (1996) propone valores hematológicos de referencia para un grupo de psitácidos donde los datos obtenidos en el presente estudio se encuentran dentro de los rangos propuestos, con excepción de los eosinófilos y monocitos. Se observan diferencias en la proporción de heterófilos y linfocitos entre las especies aviares. Por ejemplo, cotorra cubana (Amazona leucocephala) tiene de $23 \%$ a $65 \%$ de heterófilos y de $34 \%$ a $68 \%$ de linfocitos; cotorra catey (Aratinga euops) de 32\% a 65\% de heterófilos y de 31\% a 58\% de linfocitos (Soto, 2010); loro de alas naranjas del Amazonas (Amazona amazonica amazónica) tiene 61.9\% de heterófilos, y el loro frente azul (Amazona aestiva) presenta un balance entre heterófilos $y$ linfocitos (46\% y 49\%, respectivamente) (Saint-Girons \& Duguy, 1963). Similar resultado obtiene Silveira (2006) estudiando al papagayo verdadero (Amazona aestiva) (49\% y 48\%, respectivamente) y los valores reportados por Solari y colaboradores (2011) para Primolius couIoni presentaron $64.7 \%$ de heterófilos y $25.3 \%$ de linfocitos, mientras que Primolius sp. híbrido presentó $65.61 \%$ y $26.41 \%$ de heterófilos y linfocitos, respectivamente.

Se concluye que los valores hematológicos y morfológicos para Primolius sp. híbrido se encuentran dentro de los rangos reportados tanto para sus especies originarias como para otros géneros psitácidos. Por ser el primer reporte local de hibridación de estas especies, los datos obtenidos pueden ser usados como valores referenciales tanto para esta generación de híbridos como para el aporte de futuros dadores de sangre heteróloga/homóloga.

\section{Declaración de divulgación}

Los autores no informaron ningún conflicto de intereses potencial.

\section{Agradecimientos}

Agradecemos al personal de la Estación Biológica La Esmeralda (Santa Fe, Argentina) y a la Facultad de Ciencias Veterinarias (FCV) de la Universidad Nacional del Litoral (UNL, Argentina) por la orientación y asistencia durante el estudio.

\section{Referencias}

Aguilar, R., Hernández, M. \& Hernández, J. (2005). Atlas de medicina terapéutica y patología de animales exóticos. Buenos Aires: Inter Médica.

Año 15, núm. 37, noviembre 2019

ISSN: 2007-1760 (impreso) 2448-9026 (digital) | DoI: 10.30973/inventio/2019.15.37/8 
Bergman, R. (1957). The erythrocyte of snakes. Folia Haematol, 75, 92-111.

Cruz Alvarado, M., Arraga-Alvarado, C., Rincón Rincon, M., Fernández, G., Aguilar Lara, J., Villasmil-Ontiveros, Y., Gómez, O. \& Henriquez, A. (2008). Valores hematológicos de psitácidos de los géneros Ara y Amazona cautivos en zoológicos de Venezuela. Rev Cient, 18. 469-661. http://ve.scielo.org/scielo.php?script=sci arttext\&pid $=$ So798-22592008000600002

Feldman, B. \& Sink, C. (2006). Urinálise hematologia laboratorial para o clínico de pequeños animais. São Paulo: Roca.

Jordan, R. (2009). Guacamayos, una guía completa. Barcelona: Editorial Hisp Eur.

McDonald, S. (1996). The complete blood count. Avian Quarterly.

Natt, M. \& Herrick, C. A. (1952). A new diluent for counting erytrhocytes and leucocytes of the chicken. Poultry Sci, 31, 735-738.

Saint-Girons, H. \& Duguy, R. (1963). Notes de citologie sanguine comparé sur les reptiles de France. B Soc Zool Fr, 88, 613-624.

Sciabarrasi, A. \& Neme, L. (2018). El guacamayo híbrido de Santa Fe. Vet Comunicaciones. https://www.vetcomunicaciones.com.ar/page/cientifica tecnica/id/333/title/ElGuacamayo-H\%C3\%ADbrido-de-Santa-Fe-\%28Primolius-sp.\%29-originado-en-laEstaci\%C3\%B3n-Biol\%C3\%B3gica-La-Esmeralda\%2C-Santa-Fe\%2C-Argentina

Silveira, C. (2006). Valores hematológicos de referência para papagaios-verdadeiros (Amazona aestiva - Psittacidae) mantidos em cativeiro, Tesis do Mestre, Univ do Belo Horizonte.

Solari, G., David, T., Zoila, N., Rafael, A. \& José P. (2011). Parámetros hematológicos del "Guacamayo Cabeza Azul Primolius couloni" (Psittacidae) en cautiverio. Rev. Investig. Vet., 22(1). http://www.scielo.org.pe/scielo.php?pid=S1609-91172011000100004\&script=sci arttext\&tlng=en\#fo1

Soto Piñeiro, C. J., Acosta Guevara, I. \& Cruz López, E. (2009). Parámetros hematológicos de Cotorras (Amazona leucocephala) y Cateyes (Aratinga euops). REDVET. Revista Electrónica de Veterinaria, 10(7B), 1-10. https://www.redalyc.org/pdf/636/63617142007.pdf

Soto, C. (2010). Las psitácidas cubanas y su preservación. REDVET. Revista Electrónica de Veterinaria, 10(11B), 111006B ref.44. http://www.veterinaria.org/index.php/REDVET

Tell, L., Kabbur, M., Smith, W., Dahl, K. \& Cullor J. (1997). A technique for isolating heterophils from blood of Orange-Winged Amazon Parrots (Amazona amazonica amazonica). Comp Haematol, 7, 47-53. http://doi.org/10.1007/BFo1320999

Año 15, núm. 37, noviembre 2019 